\title{
Nontraumatic spinal epidural hematoma during pregnancy: diagnosis and management concerns
}

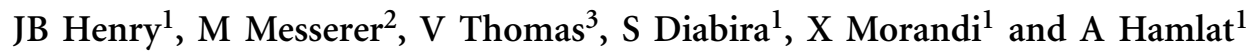

Objective: Nontraumatic spinal epidural hematoma (SEH) during pregnancy is rare. Therefore, appropriate management of this occurrence is not well defined. The aim of this study was to extensively review the literature on this subject, to propose some novel treatment guidelines.

Methods: Electronic databases, manual reviews and conference proceedings up to December 2011 were systematically reviewed. Articles were deemed eligible for inclusion in this study if they dealt with nontraumatic SEH during pregnancy. Search protocols and data were independently assessed by two authors.

Results: In all, 23 case reports were found to be appropriate for review. The mean patient age was 28 years and gestational age was 33.2 weeks. Thirteen cases presented with acute interscapular pain. The clinical picture consisted of paraplegia, which occurred approximately $63 \mathrm{~h}$ after pain onset. Spinal cord decompression was performed within an average time of $20 \mathrm{~h}$ after neurological deficit onset. Fifteen patients had cesarean deliveries, even when the gestational age was less than 36 weeks.

Conclusion: This review failed to identify articles, other than case reports, which could assist in the formation of new guidelines to treat SEH in pregnancy. However, we believe that SEH may be managed neurosurgically, without requiring prior, premature, cesarean section.

Spinal Cord (2012) 50, 655-660; doi:10.1038/sc.2012.48; published online 29 May 2012

Keywords: spinal epidural hematoma; pregnancy; diagnosis; spinal cord decompression; delivery; cesarean section

\section{INTRODUCTION}

Although spinal epidural hematoma (SEH) is a well-known, potentially devastating, neurological complication of epidural procedures, ${ }^{1,2}$ its spontaneous occurrence either in the general population, ${ }^{3}$ during pregnancy, ${ }^{4,5}$ or in the postpartum period is rare. ${ }^{6,7}$

Therefore, there is little data in the literature regarding optimal SEH management during pregnancy. Working on a case-by-case basis, a neurosurgeon must consider whether spinal cord decompression before or following delivery is best for the parturient, and an obstetrician must decide on the most appropriate method of fetus delivery.

The aim of this study was to extensively review the literature, to propose some SEH management guidelines. The goals were to ascertain the indications and contraindications of surgical and conservative management of SEH, to elucidate the most appropriate delay between diagnosis and surgery, and to identify the best surgical position and the most appropriate management for a better prognosis of both fetus and mother.

\section{MATERIALS AND METHODS}

Medline, using the PubMed interface, and Google Scholar from 1960 to December 2011 were searched. For the PubMed search, the following Medical Subject headings $(\mathrm{MeSH})$ and free text terms were applied; 8 'spinal epidural hematoma', 'pregnancy' and 'management'. No language restrictions were applied. Reference lists of identified studies and scanned abstracts from recent conference proceedings (from 2005 to 2011) were also manually reviewed. Finally, ongoing trials were investigated at clinicaltrials.gov.

Articles were eligible for inclusion in this study if they dealt with nontraumatic SEH in pregnant women. Eligibility was independently assessed, and data were independently extracted by two authors (MM and AH). Differences were resolved by consensus.

\section{RESULTS}

A total of 103 references were originally identified, from which 24 studies were initially considered as eligible. A further three articles were excluded; the first as it was the same case as reported in another study, published in another language, $, 9,10$ the second because the article presented SEH occurring after chiropractic manipulation ${ }^{11}$ and the third reported a case of unilateral epidural nerve root hematoma in a pregnant patient. ${ }^{12}$ The remaining 21 articles were all case report design; $;, 5,10,13-29$ to be noted, the article by Tada et al. ${ }^{30}$ reported 3 cases, making a total of 23 cases (Table 1).

Mean patient age at presentation was 27.7 years (range: 20-36 years). Eight women had previous pregnancies. For nine patients, it was a first-time pregnancy, and in six cases, maternity status was unknown. The mean gestational age at presentation was 33.3 weeks (range: 16-41 weeks).

One patient had been treated with heparin therapy for antiphospholipid syndrome. ${ }^{18}$ One patient presented with twin pregnancy. ${ }^{28}$ The remaining 21 patients had no significant medical history.

${ }^{1}$ Department of Neurosurgery, CHU Rennes, Hopital Pontchaillou, Rennes, France; 2Department of Neurosurgery, Centre Hospitalier Universitaire Vaudois, Lausanne, Switzerland and ${ }^{3}$ Departments of Obstetrics and Gynecology, CHU Rennes, Hopital Sud, Rennes, France 
Table 1 Clinical characteristics, surgical management and outcome of the published cases

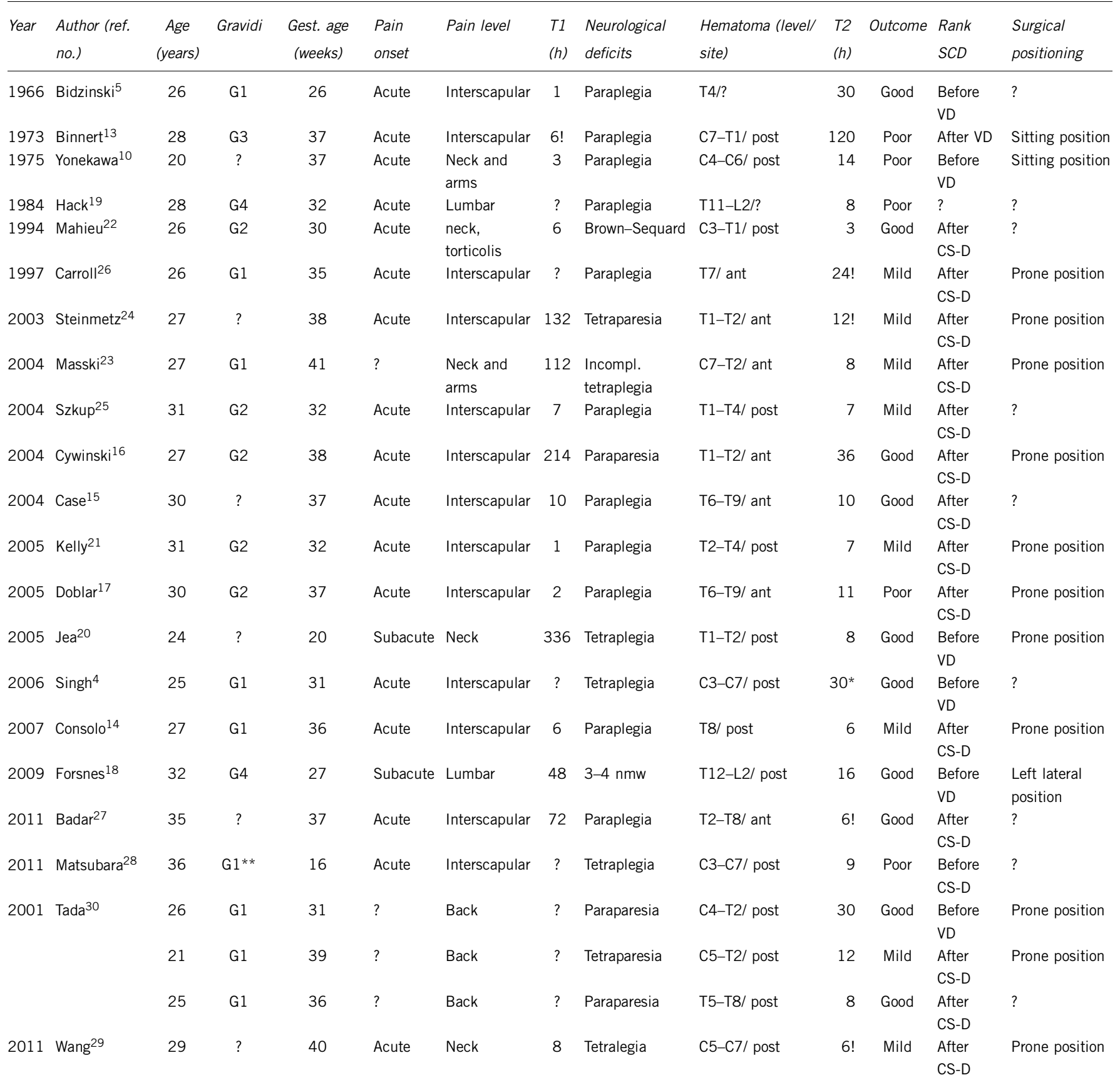

Abbreviations: Ant, anterior; C, cervical; CS-D, cesarean section delivery; G1, first gestation; Gest. age, gestational age; Good, complete recovery; Incompl., incomplete; L, lumbar; Mild, markedly improved, the patient is able to walk without assistance; Poor, the patient is not able to walk without assistance; post, posterior; Rank SCD: timing of Spinal Cord Decompression related to delivery; T, thoracic; T1, time window between onset and neurological deficits; T2, time window between neurological deficits and spinal cord decompression; VD, vaginal delivery; 3-4 nmw, third and fourth nerves motor weakness; $30^{*}, 30 \mathrm{~h}$ after onset of symptoms; **, twin pregnancy; ?, unknown; !, approximately.

Abrupt onset of pain was followed by paresthesias and either sensory and/or motor neurological deficits, within hours or days, in almost all cases. Usually, acute pain occurred within the interscapular region ( 13 out of 23 cases). The mean time period between pain onset and obvious objective neurological deficits (T1) was $60.3 \mathrm{~h}$ for 16 patients (range: $1-336 \mathrm{~h}$ ); in 7 cases no delay was cited. The mean time period between the appearance of obvious objective neurological deficits and spinal cord decompression (T2) was $18.3 \mathrm{~h}$ (range: 3-120 h) for all 23 patients. The clinical picture was incomplete or complete paraplegia in 14 cases, incomplete or complete tetraplegia in 7 cases, Brown-Séquard syndrome in 1 case and lumbar nerve motor weakness in 1 case.

The earliest case reviewed here was reported in 1966 by Bidzinskin et al. ${ }^{5}$ whereas 20 cases were published after 1980, during the era of modern imaging, including 18 diagnosed by magnetic resonance imaging (MRI). $4,14-18,20,21,23-30$

The hematoma was located in the anterior epidural space in 7 cases and the posterior epidural space in 14 cases. In two cases, the antero- 
posterior location of the hematoma was not disclosed. Three cases involved 1 vertebra, 4 cases involved 2 spinal levels, and 16 cases involved 3 or more spinal levels (range: 3-7 spinal levels; mean: 3.6 spinal levels).

In one case, ${ }^{19}$ the time of surgery (pre or postpartum) was unknown. Prepartum neurosurgery (that is, spinal cord decompression) was performed in 7 patients (6 were delivered vaginally and 1 by cesarean section).4,5,10,18,20,28,30 Additionally, in 6 of the reviewed cases, spinal cord decompression was performed initially, at a gestational age of less than 31 weeks, allowing a subsequent vaginal delivery.

In these cases, neurological symptoms included 2 paraplegias, 1 paraparesia, 3 tetraplegias and $1 \mathrm{~L} 3$ and L4 nerve root weakness. Surgical positioning was unknown for 3 cases, sitting position was used in 1 case, prone position in 2 cases and the left lateral position in 1 case. Surgery outcome noticed complete recovery in 5 out of the 7 cases. Postpartum neurosurgery was performed in 15 patients and in 1 case, the timing of neurosurgery, in relation to delivery, was not detailed. In 14 out of these 15 patients, a cesarean section delivery preceded spinal cord decompression, even in cases with a gestational age of less than 36 weeks (range 30-41 weeks). One patient was vaginally delivered before surgery. In these cases, neurological symptoms included 8 paraplegias, 2 paraparesis, 1 Brown-Séquard syndrome, 1 tetraplegia and 3 tetraparesis. Surgical positioning was unknown in 5 cases, sitting position was used in 1 case and prone position in the remaining 9 cases. Surgery outcome noticed complete recovery in 5 out of 11 cases, markedly improvement in 8 cases and no evolution (patients were not able to walk without assistance) in 2 cases.

Most patients underwent laminectomy surgery, whereas a hemilaminectomy was performed on one patient, ${ }^{26}$ and laminoplasty on another patient. $^{28}$

No fetal death was reported, and none of the patients treated neurosurgically before childbirth experienced preterm labor.

At follow-up reviews, 10 patients showed total neurological recovery, with independent ambulation. Mild recovery, with residual deficits, which interfered, to some degree, with daily life, was reported in 8 patients (follow-up range: 3 days-10 months). Five patients had several disabling sequelae (follow-up range: 45 days-11 months). However, most of the published cases did not have a long-term follow-up (mean: 5 months; range: 3 days-1 year).

\section{DISCUSSION}

In our literature review, we found 23 cases $^{4,5,10,13-30}$ of SEH occurring during pregnancy. Clinical presentation and symptoms are similar in both gravid and non-gravid patients. The archetypal clinical presentation is an abrupt onset of severe spinal pain. ${ }^{3,31,32}$ This sentinel symptom is present in $80 \%$ of SEH cases in the general population ${ }^{3}$ and $89 \%$ of SEH cases in pregnant women (17 out of 19).

Usually, in pregnant patients, pain occurs spontaneously or in the course of daily, slightly strenuous, events. ${ }^{10,13,21}$ According to the published cases, acute spinal pain was located mostly in the interscapular region. It was a novel symptom in almost all parturient and none mentioned an antecedent of spinal pain. However, it is unclear if some similar patient history has been omitted from clinical reports.

Cervical and cervico-thoracic SEH can give rise to acute cervical or interscapular pain. Hematomas located at the midthoracic level present with thoracic or chest pain, ${ }^{5,21}$ whereas thoracolumbar hematomas usually present with acute back pain. ${ }^{18,19,30}$ The pain may irradiate to the limbs in a radicular fashion, as a result of nerve root compression, if the hematoma extends ${ }^{18}$ or develops laterally. ${ }^{12}$
Hematoma development in the lower thoracic or lumbar region may result in pain, which can irradiate to the buttocks and the lower extremities, and the clinical picture could be that of cauda equina syndrome, as reported in non-pregnant patients. ${ }^{33,34}$

Neurological signs and symptoms develop after a free interval of hours, days or even weeks during which time the pain main either slowly subside, or persist. Spreading numbness and weakness herald the progressive loss of sensory function and motor deficit, below the segmental site of the hematoma. This may progress more or less rapidly to full paraplegia, or tetraplegia with sphincter incontinence, and, in exceptional instances, to Brown-Séquard syndrome. ${ }^{22}$

The development of neurological deficits confirms the diagnosis, although missed diagnoses have been reported. ${ }^{6,13,17,18,23}$ Therefore, prompt recognition of the symptoms, before the onset of severe neurological deficits, is important to avoid permanent disabling sequelae. The median time period between pain onset and neurological deficits was $7.5 \mathrm{~h}$ in the reviewed cases.

Acute spinal pain in pregnant women, without neurological deficit, is often considered inconsequential by physician and pain subsidence may inadvertently validate the diagnosis of more common and less serious conditions, such as general spinal pain related to pregnancy. However, pregnancy-related spinal pain is normally lumbar spinal pain, which usually begins early in the course of pregnancy, and has neither an acute onset nor is associated with any objective or subjective neurological deficit. Acute spinal pain, associated with subjective or objective neurological deficits, should alert the physician, as this clinical picture may reveal either SEH or other spinal conditions. ${ }^{35-37}$

The pathophysiology of SEH is still unclear and the source of bleeding is debated. ${ }^{38,39}$ The vertebral venous system may be considered as a valveless anatomical system located in a negative pressure compartment (that is, the epidural space). In this negative pressure compartment, blood is supposed to flow in any direction depending on the changes of the thoracoabdominal pressure and hydrostatic factors. The internal resistance of the epidural venous system is correlated with the direction of blood flow, the epidural pressure and the pressure gradient between intradural and thoracoabdominal veins that drain the epidural veins. It has been postulated that an acute increase of the spinal epidural vein pressure is a probable contributing factor ${ }^{40}$ and that epidural veins are more susceptible to congestion during pregnancy. ${ }^{15,25}$ In addition, aortocaval compression may significantly impede venous return as early as the middle of the second trimester, and may increase spinal vein congestion within the negative pressure compartment of the epidural space. ${ }^{15,25}$ However, such phenomena are common in pregnancy, whereas pregnancy-related SEH is a rare occurrence. It has also been suggested that predisposing anatomical factors, such as abnormal vein development, areas of locus minoris resistentiae (consisting of a network of weakened epidural veins), ${ }^{10,26,39}$ structural vessel changes induced by estrogen and progesterone excess during the third trimester, ${ }^{16}$ along with various other triggering and/or influencing factors $3,40,41$ may favor $\mathrm{SEH}$ occurrences. Therefore, it may be surmised that pregnancy is neither necessary nor sufficient to cause SEH, although it may be a contributory cause in some particular instances. We assume that nontraumatic SEH is a multi-factorial dynamic condition. ${ }^{31}$ Each isolated factor cannot be the single cause of nontraumatic SEH in any patient. Several of these factors must interact to cause SEH. Additionally, usually it is difficult to determine the chronological order of occurrence of each contributing factor in nontraumatic SEH development. $^{3}$ 
Any clinical suspicion of SEH requires urgent assessment ${ }^{31}$ and diagnostic confirmation can be achieved with MRI. As there are no reported adverse effects of MRI on fetal growth and development, ${ }^{42}$ this investigative imaging should be used in place of computed tomography scanning, using ionizing radiation imaging.

The MRI features of acute SEH are relatively specific. Acute SEH is an iso to hyper-intense dorsal convex lens-shaped lesion on T1-weighted images and hyper-intense on T2 weighted images. An increased signal intensity on both $\mathrm{T} 1$ and $\mathrm{T} 2$ weighted images is seen in subacute SEH cases. ${ }^{34}$ Furthermore, SEH is most often located in the posterior part of the cervical or cervico-thoracic region and it may extend over multiple spinal levels.

The optimal diagnostic and therapeutic strategies in pregnant women are matters of debate, as the patient and the unborn child must be considered. The acute presentation of SEH, the degree of decline of maternal neurological function, and gestational age add further complexity to timing and surgical considerations. Therefore, the goal of successful therapeutic management is to prevent the development of maternal disabilities, usually associated with SEH, and to preserve fetal health. Thus for each case, management strategies must consider the patient's neurological status and the gestational age. Furthermore the surgical risks for the fetus should be weighed up against the potential severity of the neurological deficits of the mother.

Based on a review of the cases available, surgical treatment does appear to be a trend in the management of SEH during pregnancy. Treatment of SEH in these cases have included either induction of preterm delivery followed by neurosurgery, or prepartum neurosurgery, but rarely conservative management. ${ }^{11}$

As the traditional strategy of unconditional, urgent, neurosurgical decompression is inappropriate in some cases, owing to some spontaneous recovery in pregnant ${ }^{11}$ and non-pregnant patients, ${ }^{33,40,43,44}$ the conservative management of SEH must also be considered as a potentially viable management technique.

Conservative management has previously been considered in nonpregnant patients with either no or mild neurological deficits and those who show rapid clinical improvement. However, if the improvement does not progress, or further neurological deterioration occurs, surgery is recommended. ${ }^{40,43-45}$ The indications for this expectant treatment are not well defined. There are no predictive characteristics that allow a definitive segregation between conservative and surgical treatment. ${ }^{33,46-48}$ It is difficult to determine how mild the neurological status should be ${ }^{49}$ or how long the appropriate improvement time period, to warrant the continued use of conservative treatment. ${ }^{43,44,50}$ Moreover, it is impossible to predict the degree of spontaneous neurological recovery.

Nonetheless, when the spinal cord has minimal radiological compression and the patient presents only with pain and paresthesia, observation alone with close clinical and radiological follow-up, may be sufficient. However, it must be emphasized that neurological monitoring for an extended period in a hospital with neurosurgical and MRI departments is mandatory for conservative treatment.

Corticosteroid therapy is reported as an adjunct therapy before $e^{43,48}$ or following spinal cord decompression ${ }^{51,52}$ in non-pregnant patients, although there is no evidence to support this statement. Corticosteroids cross the placenta and help to promote fetal maturation, particularly lung maturation. ${ }^{42}$ However they should be administered for short periods only, owing to the risk of induced adrenal insufficiency of the fetus. ${ }^{53}$

Spinal cord decompression should be performed in those patients with severe neurological deficits and those with mild neurological deficits, that do not improve rapidly or that worsen. In a study of 330
SHE in non-pregnant patients, Groen et al. ${ }^{41}$ showed that treatment outcome was favorable and recovery was significantly improved when decompression was performed within $36 \mathrm{~h}$ in patients with complete deficits and within $48 \mathrm{~h}$ in patients with incomplete deficits. Neurosurgical decompression is also indicated in cases with hematomas, which extend above $\mathrm{C} 5$, particularly as the mortality risk is reported to be $16-30 \%{ }^{38,41}$ in non-pregnant patients.

Obstetric management depends on both the pregnancy term and the necessity of spinal cord decompression.

In 14 out of 23 pregnant patients, a cesarean section delivery preceded spinal cord decompression, even in cases with a gestational age of less than 36 weeks (range 30-41 weeks). The gestational age was 36 weeks or more in 4 of these cases. It may be speculated that this management was either mandatory or was an ancient routine practice, retained to avoid the anxiety and stress that may result from attempting to balance the risks and benefits of simple spinal cord decompression.

We believe that SEH could be managed surgically, without any need to perform premature cesarean delivery of the fetus, ${ }^{10}$ because there is no evidence of preterm labor following spine surgery. Furthermore, the morbidity and mortality risks for both the pregnant patient and fetus, as a result of neurosurgery, are less than the mother's risk of permanent neurological deficits due to delayed spinal cord decompression. Additionally, in six of the reviewed cases, spinal cord decompression was performed initially, at a gestational age of less than 31 weeks, allowing a subsequent vaginal delivery.

Therefore, when clinical evidence suggests the need for an urgent or emergent operation in a pregnant patient, the pregnancy should not affect the decision to proceed, especially in the presence of disabling neurological symptoms. Prepartum spinal cord decompression can be performed safely. Reducing the delay to spinal cord decompression is likely to improve functional status, maintain a healthy pregnancy and probably allow a vaginal delivery. $4,5,10,18,20,30$

It is our belief that cesarean section should be performed only if there is preterm labor or a risk of harm to the fetus. In these cases, delivery of the fetus by cesarean section, instead of vaginal delivery is preferable as it minimizes delay for spinal cord decompression. Furthermore, vaginal delivery prior to surgical decompression is risky, due to unpredictable labor duration ${ }^{16}$ and increase in abdominal pressure. The combination of the cesarean section and the spinal cord decompression, or simple spinal cord decompression, makes general anesthesia the anesthetic technique of choice.

However, this supposition, regarding management of SEH in pregnancy, requires further reflection and contribution from obstetricians, neurosurgeons and anesthesiologists, and may be relevant to the management of other pregnancy-related diseases.

From a neurosurgical point of view, spine surgery, performed on a pregnant patient, needs special consideration in terms of positioning. Several surgical positions have been used including prone, sitting and lateral positions. We concur with Jea et al. ${ }^{20}$ that a prone position is preferable. When compared with the sitting or lateral position, prone positioning, which involves positioning the pregnant patient on four frames (L-shaped form), sufficiently high enough to hang the protuberant abdomen, encourages the uterus to migrate to the large maternal vessels and relieves uterine compression. ${ }^{20}$ The clavicles and the anterosuperior iliac spine support both the chest and abdomen that rest laterally on the frames, whereas the head is fixed, in a straight position, by a three-point Mayfield holder. Most patients were managed by laminectomy, with one case of a hemi-laminectomy. We believe that laminectomy is not always necessary for SEH removal, as a hemi-laminectomy, an inter-laminar approach, a laminoplasty and a uni $^{4}$ or bilateral alternating hemi-laminectomy may be sufficient. 
From an obstetrical point of view, although no untoward harm, either to the fetuses or newborns has been reported in these reviewed cases, both should be monitored closely. After 23 weeks gestation, fetal heart monitoring during neurosurgery, is indicated to highlight any abdominal heart rate patterns, which may alert the anesthesiologist, obstetrician and neurosurgeon to take appropriate action to safeguard the fetus.

The two strongest predictors of outcome, identified up to now in non-pregnant patients, are the severity of preoperative deficits and the time interval between onset of symptoms and spinal cord decompression. ${ }^{40,41,50}$ Although the follow-up time duration was too short (mean 5 months) to draw any meaningful conclusions, the prognosis of pregnancy-related SEH seems similar to that of non-pregnant patients.

\section{Limits of the study}

A major limitation of this study is that, despite extensive research, only case reports were found that could be considered eligible for consideration. No level A, evidence-based, medical studies exist to support an official recommendation or guidelines for managing nontraumatic spontaneous SEH during pregnancy.

\section{CONCLUSION}

$\mathrm{SEH}$ is a rare condition, particularly among pregnant women. Acute back pain should warrant clinical suspicion, with diagnostic confirmation being achieved with MRI. Successful management requires close cooperation between neurosurgeons, obstetricians and anesthesiologists. If there are neurological symptoms, surgery is immediately required, before fetal delivery.

\section{CONFLICT OF INTEREST}

The authors declare no conflict of interest.

\section{ACKNOWLEDGEMENTS}

For help with editing in English, and other valuable advice, we thank Dr Conor Moran of the National University of Ireland Galway and Professor A Elghalem of the University of Algiers.

1 Lang SA. Spinal epidural hematoma: still an enigma. J Clin Anesth 2004; 16 : 317-319.

2 Sage DJ. Epidurals, spinals and bleeding disorders in pregnancy: a review. Anaesth Intensive Care 1990; 18: 319-326.

3 Kreppel D, Antoniadis G, Seeling W. Spinal hematoma: a literature survey with metaanalysis of 613 patients. Neurosurg Rev 2003; 26: 1-49.

4 Singh DP, Lamtha SC, Kumar S. Spontaneous spinal epidural haematoma during pregnancy. J Assoc Physicians India 2009; 57: 540-542.

5 Bidzinski J. Spontaneous spinal epidural hematoma during pregnancy. Case report. J Neurosurg. 1966; 24: 1017

6 Bose S, Ali Z, Rath GP, Prabhakar H. Spontaneous spinal epidural haematoma: a rare cause of quadriplegia in the post-partum period. Br J Anaesth 2007; 99: 855-857

7 Mechurova A, Chrobok J, Prochazka V, Prochazka M. Spontaneous spinal epidural haematoma at C5-Th7 level after spontaneous delivery. Case report. Rivista Neuroradiol 2006; 19: 169-175.

8 Kassai B, Sonie S, Shah NR, Boissel JP. Literature search parameters marginally improved the pooled estimate accuracy for ultrasound in detecting deep venous thrombosis. J Clin Epidemiol 2006: 59: 710-714.

9 Nishikawa M, Mehdorn HM, Yonekawa Y. Spontaneous spinal epidural hematoma. No Shinkei Geka 1976; 4: 685-688.

10 Yonekawa Y, Mehdorn HM, Nishikawa M. Spontaneous spinal epidural hematoma during pregnancy. Surg Neurol 1975; 3: 327-328.

11 Heiner JD. Cervical epidural hematoma after chiropractic spinal manipulation. Am J Emerg Med 2009; 27: e1021-e1022.

12 Kuczkowski KM, Benumof JL, Reisner LS, Alfery D, Myers R. Unilateral epidural nerve root hematoma in a parturient. Acta Anaesthesiol Belg 2003; 54: 157-159.
13 Binnert D, Thierry A, Michiels R, Soichot P, Perrin M. Presentation of a new case of spontaneous spinal extradural hematoma observed during labor. J Med Lyon 1971; 52 : 1307-1309.

14 Consolo D, Vadala AA, Rollin P, Merle B, Girard C. Spontaneous spinal epidural haematoma during pregnancy. Ann Fr Anesth Reanim 2007; 26: 455-458.

15 Case AS, Ramsey PS. Spontaneous epidural hematoma of the spine in pregnancy. Am J Obstet Gynecol 2005; 193: 875-877.

16 Cywinski JB, Parker BM, Lozada LJ. Spontaneous spinal epidural hematoma in a pregnant patient. J Clin Anesth 2004; 16: 371-375.

17 Doblar DD, Schumacher SD. Spontaneous acute thoracic epidural hematoma causing paraplegia in a patient with severe preeclampsia in early labor. Int $\mathrm{J}$ Obstet Anesth 2005; 14: 256-260.

18 Forsnes E, Occhino A, Acosta R. Spontaneous spinal epidural hematoma in pregnancy associated with using low molecular weight heparin. Obstet Gynecol 2009; 113: 532-533.

19 Hack I, Cademartori MS, Mamani RS, Beltrame CM, Cademartori CG. Spontaneous spinal epidural hematoma during pregnancy: report of a case. Arq Neuropsiquiatr 1984; 42: 41-44.

20 Jea A, Moza K, Levi AD, Vanni S. Spontaneous spinal epidural hematoma during pregnancy: case report and literature review. Neurosurgery 2005; 56: E1156.

21 Kelly ME, Beavis RC, Hattingh S. Spontaneous spinal epidural hematoma during pregnancy. Can J Neurol Sci 2005; 32: 361-365.

22 Mahieu X, Kridelka F, Pintiaux A, Hans P, Brichant JF, Born J et al. Spontaneous cervical extradural hematoma in a pregnant woman. J Gynecol Obstet Biol Reprod 1994; 23: 99-102

23 Masski G, Housni B, Ibahiouin K, Miguil M. Spontaneous cervical epidural haematoma during pregnancy. Int J Obstet Anesth 2004; 13: 103-106.

24 Steinmetz MP, Kalfas IH, Willis B, Chalavi A, Harlan RC. Successful surgical management of a case of spontaneous epidural hematoma of the spine during pregnancy. Spine J 2003; 3: 539-542.

25 Szkup P, Stoneham G. Case report: spontaneous spinal epidural haematoma during pregnancy: case report and review of the literature. Br J Radiol 2004; 77: 881-884.

26 Carroll SG, Malhotra R, Eustace D, Sharr M, Morcos S. Spontaneous spinal extradural hematoma during pregnancy. J Matern Fetal Med 1997; 6: 218-219.

27 Badar F, Kirmani S, Rashid M, Azfar SF, Yasmeen S, Ullah E. Spontaneous spinal epidural hematoma during pregnancy: a rare obstetric emergency. Emerg Radiol 2011; 18: 433-436.

28 Matsubara S, Inoue H, Takamura K, Kimura A, Okuno S, Fujita A et al. Spontaneous spinal epidural hematoma at the 16 th week of a twin pregnancy. J Obstet Gynaecol Res 2011; 37: 1466-1469.

29 Wang P, Xin XT, Lan H, Chen C, Liu B. Spontaneous cervical epidural hematoma during pregnancy: case report and literature review. Eur Spine J 2011; 20(Suppl 2): S176-S179.

30 Tada S, Yasue A, Nishizawa H, Sekiya T, Hirota Y, Udagawa Y. Spontaneous spinal epidural hematoma during pregnancy: three case reports. J Obstet Gynaecol Res 2011; 37: $1734-1738$.

31 Messerer M, Dubourg J, Diabira S, Robert T, Hamlat A. Spinal epidural hematoma: not always an obvious diagnosis. Eur J Emerg Med 2012; 19: 2-8.

32 Bruyn G, Bosma L. Handbook of Clinical Neurology. Co NHP (ed.). Amsterdam, New York Norrh Holland Pub. Co, 1976.

33 Park HJ, Kim IS, Lee SW, Sung JH, Hong JT. Spontaneous resolution of spinal epidural hematoma: a case report. Kor J Spine 2008; 5: 107-109.

34 Jamjoom ZA. Acute spontaneous spinal epidural hematoma: the influence of magnetic resonance imaging on diagnosis and treatment. Surg Neurol 1996; 46: 345-349.

35 Castel E, Lazennec JY, Chiras J, Enkaoua E, Saillant G. Acute spinal cord compression due to intraspinal bleeding from a vertebral hemangioma: two case-reports. Eur Spine J 1999; 8: 244-248.

36 Bui QH, Luu DH, Nguyen QK. Haematomyelia during pregnancy: pathogenic discussion. First observations. Proc Aust Assoc Neurol 1968; 5: 515-519.

37 Schwenkreis P, Pennekamp W, Tegenthoff M. Differential diagnosis of acute and subacute non-traumatic paraplegia. Dtsch Arzteb/ 2006; 103: 2948-2954.

38 Beatty RM, Winston KR. Spontaneous cervical epidural hematoma. A consideration of etiology. J Neurosurg 1984; 61: 143-148.

39 Groen RJ, Ponssen H. The spontaneous spinal epidural hematoma. A study of the etiology. J Neurol Sci 1990; 98: 121-138.

40 Groen RJ. Non-operative treatment of spontaneous spinal epidural hematomas: a review of the literature and a comparison with operative cases. Acta Neurochir 2004; 146: 103-110.

41 Groen RJ, van Alphen HA. Operative treatment of spontaneous spinal epidural hematomas: a study of the factors determining postoperative outcome. Neurosurgery 1996; 39: 494-508; discussion 508-499.

42 Delisle MF, Valimohamed F, Money D, Douglas MJ. Central nervous system complications of von Hippel-Lindau disease and pregnancy; perinatal considerations: case report and literature review. J Matern Fetal Med 2000; 9: 242-247.

43 Kato S, Seki H, Koshu K. Acute cervical spinal epidural hematoma with spontaneous resolution-case report. Neurol Med Chir 1994; 34: 23-26.

44 Wagner S, Forsting M, Hacke W. Spontaneous resolution of a large spinal epidural hematoma: case report. Neurosurgery 1996; 38: 816-818.

45 Kinoshita M, Asai A, Komeda S, Yoshimura K, Takeda J, Uesaka T et al. Spontaneous regression of a spinal extradural arteriovenous fistula after delivery by cesarean section. Neurol Med Chir 2009; 49: 313-315.

46 Fukui MB, Swarnkar AS, Williams RL. Acute spontaneous spinal epidural hematomas. Am J Neuroradiol 1999; 20: 1365-1372. 
47 Matsumura A, Namikawa T, Hashimoto R, Okamoto T, Yanagida I, Hoshi M et al. Clinical management for spontaneous spinal epidural hematoma: diagnosis and treatment. Spine J 2008; 8: 534-537.

48 Sinclair AJ, Carroll C, Davies B. Cauda equina syndrome following a lumbar puncture. J Clin Neurosci 2009; 16: 714-716.

49 Wong YW, Luk KD. Spinal epidural hematoma in a scoliotic patient with long fusion: a case report. Spine J 2008; 8: 538-543.

50 Lawton MT, Porter RW, Heiserman JE, Jacobowitz R, Sonntag VK, Dickman CA Surgical management of spinal epidural hematoma: relationship between surgical timing and neurological outcome. J Neurosurg 1995; 83: 1-7.
51 Omori N, Takada E, Narai H, Tanaka T, Abe K, Manabe Y. Spontaneous cervical epidural hematoma treated by the combination of surgical evacuation and steroid pulse therapy. Intern Med 2008; 47: 437-440.

52 Hancock JB, Field EM, Gadam R. Spinal epidural hematoma progressing to Brown-Sequard syndrome: report of a case. J Emerg Med 1997; 15 309-312.

53 Vougioukas VI, Kyroussis G, Glasker S, Tatagiba M, Scheufler KM. Neurosurgical interventions during pregnancy and the puerperium: clinical considerations and management. Acta Neurochir 2004; 146: 1287-1291; discussion 1291-1292. 